\section{Categorías, esquemas y sombras: El caso del fideicomiso peruano ${ }^{(*)}$}

Freddy Escobar Rozas

Abogado por la Pontificia Universidad Católica del Perú. Master en Derecho (Harvard Law School). Profesor de Análisis Económico del Derecho,

Contratos, Mercado e Instituciones Legales, y Teoría Legal en la Pontificia Universidad Católica del Perú. Miembro del Consejo Consultivo de la Revista ADVOCATUS.

\section{Guillermo Cabieses Crovetto}

Abogado por la Universidad de Lima. Master en Derecho (University of Chicago Law School). Profesor de Análisis Económico del Derecho, Economía, Contratos y Teoría Legal en la Pontificia - Universidad Católica del Pérú, Universidad de Lima y Universidad Peruana de

Ciencias Aplicadas.

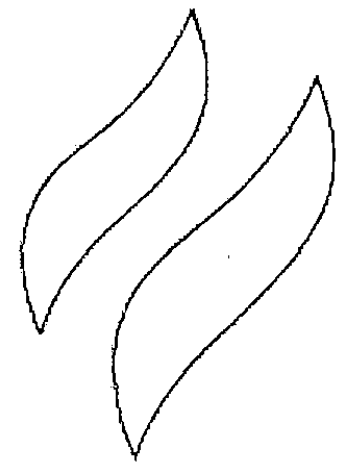

Miembro y Ex-Director de Eventos de la Revista ADVOCATUS.

SUMARIO:

I. Introducción.

II. Categorías y esquemas:

1. Definiciones;

2. Esquemas individuales (Self Schemas);

3. Esquemas sobre Personas (Person Schemas);

4. Esquemas sobre Roles (Role Schemas);

5. Esquemas sobre Eventos (Event Schemas);

6. Paradojas.

III. Conceptos legales.

IV. Noción de derecho.

V. Funciones del fideicomiso.

VI. Dominio fiduciario en el sistema legal peruano.

VII. Reconociendo la realidad.

VIII. Conclusión. 
"The law cannot be dealt with as if it contained only the axioms and corollaries of a book of mathematics" Oliver Wendel Holmes (1881)

\section{INTRODUCCIÓN}

El 27 de noviembre de 2008 el Diario Oficial El Peruano publicó la Resolución No. 316-2008SUNARP/SN, aprobada por la Superintendencia Nacional de Registros Públicos con el fin de regular la inscripción del fideicomiso. La decisión de eliminar los vacíos y resolver las controversias existentes en torno a la forma de inscripción del fideicomiso es, qué duda cabe, loable, desde que la incertidumbre eleva los costos de transacción del mercado.

No obstante ello, llama poderosamente nuestra atención la tipificación que la referida resolución otorga al dominio fiduciario. En efecto, en su Sección Antecedentes y Consideraciones la norma en cuestión señala que si bien el dominio fiduciario no es un derecho de propiedad en los términos definidos por el artículo 923 del Código Civil, sí es un derecho real de titularidad del fiduciario.' En nuestra opinión, esta tipificación es errónea desde todo punto de vista, pues, además de contradecir abiertamente el concepto filosófico y legal de "derecho", ignora la función contemporánea que el fideicomiso cumple como "forma organizacional". El error en la tipificación del dominio fiduciario genera consecuencias nocivas, al impedir que preste- mos atención a los problemas más importantes que el fideicomiso presenta, como el de las "manos muertas" y el de los estándares de diligencia aplicables al fiduciario.

En las líneas que siguen justificaremos estas afirmaciones. Para tal fin, trataremos previamente los siguientes tópicos: (a) el rol que cumplen las categorias y los esquemas dentro del proceso cognitivo; (b) el papel obstructivo que juegan los conceptos legales en el Civil Law; (c) la noción filosófica y legal de "derecho"; $y,(d)$ las diversas funciones del fideicomiso, en especial la organizacional.

\section{CATEGORÍAS Y ESQUEMAS}

Las categorías y los esquemas son las piedras angulares de la estructura del fenómeno cognitivo. ${ }^{4}$ En efecto, los seres humanos nos valemos de categorías y esquemas para procesar la enorme cantidad de información que nos rodea. Las categorías y los esquemas influencian poderosamente nuestra actividad cotidiana y nuestra capacidad cognitiva, determinando no solamente qué información llamará nuestra atención, sino también cómo la organizaremos, qué inferencias extraeremos de ella y qué cosas recordaremos u olvidaremos.

La ausencia de categorías y conceptos claros genera el incremento del esfuerzo que las personas deben realizar para procesar cognitivamente la información que reciben y, por ende, desincentiva el aprendizaje de nuevas ideas y el procesamiento de nuevas experiencias. ${ }^{6}$ Ello

1 Textualmente: "[l] a transferencia de los bienes en fideicomiso no es en propiedad, al menos no en los términos previstos en el artículo 923 del Código Civil, sino en dominio fiduciario. Sin embargo, tal dominio fiduciario viene a constituir un derecho real a favor del fiduciario (...)".

2 El problema de las manos muertas está referido al control que las personas ejercen sobre los recursos aun después de fallecer.

3 El problema de los costos de agencia está referido al monitoreo de las actividades de quienes trabajan para nosotros. Usuaimente, ese monitoreo no puede realizarse en términos óptimos por los altos costos que tal cosa supone. Por tal motivo, quienes trabajan para nosotros normalmente tienen incentivos para defraudarnos.

4 CHEN, Ronald y HANSON, Jon, Categorically Biased: The Influence of Knowledge Structures on Law and Legal Theory. En: Southern California Law Review, Vol. 77, 2004, p. 1131.

5 lbídem.

6 Ibidem, p. 1132. 
porque sencillamente procesar información nueva es una actividad relativamente costosa, al demandar mayor concentración, tiempo, imaginación, etc.

\section{Definiciones}

Una categoría es el resultado de un proceso de clasificación (grupal) de elementos, experiencias, ejemplos o argumentos; mientras que un esquema es un conjunto organizado de conocimientos sobre una categoría.?

Como afirman Chen y Hanson, la categorización (o el uso de categorías) deriva en la clasificación de la información sobre la experiencia, mientras que la esquematización deriva en el conocimiento desarrollado sobre la experiencia. Este conocimiento nos permite extraer inferencias o derivar predicciones. ${ }^{8}$

En términos comparativos, mientras las categorías constituyen las "cajas" en las cuales "colocamos" o "guardamos" la información que nos rodea, los esquemas constituyen los cuerpos de conocimiento que hemos desarrollado sobre el contenido de esas cajas y nos sirven para poder inferir o predecir situaciones, conductas o resultados. ${ }^{9}$

Así entendidos, los esquemas sirven para responder las preguntas “¿qué es esto?", “¿cómo funciona esto?", "¿por qué está aquí?", "¿qué hace?", entre otras. ${ }^{10}$ Como explican Augoustinos y Walter, los esquemas son conceptualizados como estructuras mentales que contienen expectativas y conocimientos generales acerca del mundo ${ }^{11}$, por lo que sirven para que los individuos seleccionen y procesen la información que reciben de su ambiente social. ${ }^{12}$ En razón de ello, los esquemas guían lo que nos interesa, lo que percibimos, lo que recordaremos y lo que inferimos. ${ }^{13}$

La literatura psicológica ha clasificado los esquemas que normalmente usamos de la siguiente manera: "esquemas individuales" (self schemas), "esquemas sobre personas" (person schemas), "esquemas sobre roles" (role schemas) y "esquemas de eventos" (event schemas). A continuación, explicamos brevemente en qué consisten cada uno de ellos.

\section{Esquemas Individuales (Self Schemas)}

Los "esquemas individuales" contienen el conocimiento que hemos desarrollado sobre nosotros mismos, incluyendo nuestras características, tendencias y disposiciones. ${ }^{14}$ Los individuos podemos ser "esquemáticos"o"aesquemáticos" respecto de las características humanas. ${ }^{15}$ Así, somos "esquemáticos" con relación a las características que consideramos importantes para nosotros y que entendemos poseer de alguna

\section{Ibidem.}

8 Ibídem.

9 Como afirman Shelley TAYLOR y Jennifer CROCKER, "(...) a cognitive structure that consists in part of the representation of some defined stimulus domain. The schema contains general knowledge about the domain, including a specification of the relationships among its attributes, as well as specific examples or instances of the stimulus domain. As such, one of the chief functions of a schema is to provide an answer to the question "what is it". The schema provides hypotheses about incoming stimuli, which include plans for interpreting and gathering schema-related information". Ver: Schematic Biased Bases of Social Information Processing. En: Social Cognition: The Ontario Symposium, Editores: TORY HIGGIND E. y otros, Lawrence Erlbaum Assoc Inc., New Jersey, 1981, Vol. 1, p. 91.

10 CHEN, Ronald y Jon HANSON, Op. cit., p. 1133.

11 AUGOUSTINOS, Martha e WALKER, lain, Social Cognition: An Integrated Introduction, SAGE Publications, London, 1995, p. 34.

12 Ibídem.

13 Ibidem.

14 Ibídem, p. 1134.

15 lbídem. 
forma. ${ }^{16}$ De otro lado, somos "aesquemáticos" con relación a las caracteristicas que consideramos poco representativas para nosotros y que entendemos no resultan descriptivas de nuestra realidad personal. ${ }^{17}$

Los esquemas materia de análisis abarcan un rango sumamente amplio de nuestra personalidad: desde las etiquetas ideológicas que tenemos (tales como liberales o conservadores) hasta distinciones que realizamos comúnmente de acuerdo a razas, géneros y preferencias sexuales. ${ }^{18}$

Un ejemplo dramático de "esquemas individuales" lo encontramos en el caso de los soldados de los ejércitos de Hitler. Estos solados podrían haber sido definidos como "esquemáticos" con relación al "patriotismo", pero "aesquemáticos" con relación al judaísmo, debido a que se veían en una categoría y no en la otra. ${ }^{19}$ Estos dos "esquemas individuales" nos demuestran que no debemos confiar necesariamente en su valor moral como guía de conducta. ${ }^{20}$

\section{Esquemas sobre Personas (Person Schemas)}

Los "esquemas sobre personas" representan las estructuras de conocimiento que hemos desarrollado sobre otros individuos, incluyendo sus características, comportamientos y metas. ${ }^{21}$
Nosotros utilizamos estos esquemas como una guía para realizar categorizaciones, inferencias y procesos de memoria respecto de las personas que encontramos.22

Dos de los "esquemas sobre personas" más populares son los "esquemas sobre características" y los "esquemas sobre metas": 23 Los primeros se enfocan en la clasificación de las personas sobre la base de sus características y de las inferencias que de aquellas podemos realizar. ${ }^{24}$ Los segundos, por su parte, se enfocan en la clasificación de las personas sobre la base de lo que sabemos sobre sus metas u objetivos. ${ }^{25}$

Los "esquemas sobre características" son empleados para describir ingredientes importantes acerca de la personalidad de los demás. Esa descripción influencia nuestra evaluación sobre las personas y guía nuestras decisiones acerca de cómo interactuar con los demás. ${ }^{26}$

Un ejemplo de cómo funcionan estos esquemas lo encontramos en el caso de las citas a ciegas. Como demuestran los experimentos realizados, normalmente somos más aptos a aceptar una cita a ciegas con alguien que es descrito como atractivo, amigable e inteligente, que con alguien que es descrito como dominante, serio y provincial. ${ }^{27}$ Incluso en la cita a ciegas somos más propensos a ver a la otra persona de la manera en la que fue descrita previamente a

16 Ibidem.

17 CHEN, Ronald y HANSON, Jon, Op. Cit., p. 1134.

18 Ibidem.

19 Ibidem.

20 Ibídem.

21 Ibidem, p. 1135.

22 lbídem.

23 Ibidem.

24 lbídem.

25 Ibídem, p. 136

26 WYER, Robert S. y LAMBERT, Alan J., The Role of Trait Constructs in Person Perception: An Historical Perspective. En: So cial Cognition: Impact on Social Psychology, Editor: DEVINE, Patricia y otros, Academic Press, New York, 1994, p. 109.

27 Ibidem. 
la cita, es decir, tratamos de buscar en ella las características que nos fueron comentadas y veremos en sus actos o gestos rasgos de esas características, así no las tenga. Esto ocurre debido a que nuestras expectativas dirigen nuestra atención, en ese momento y en retrospectiva, hacia los comportamientos de la persona con la que se tiene la cita que denotan esos atributos. ${ }^{28}$ No obstante, la mayoría de las citas a ciegas terminan siendo una mala experiencia, a pesar de las descripciones previas favorables que se puedan haber recibido. ${ }^{29}$ De hecho, si las personas fueran en efecto atractivas, simpáticas e inteligentes, no requerirían de citas a ciegas.

Los "esquemas sobre metas", por su parte, son empleados para guiar nuestras categorizaciones, memorias e inferencias sobre la base del conocimiento que tenemos acerca de las metas de los demás.

Un ejemplo de cómo funcionan estos esquemas lo encontramos en el siguiente experimento. Un estudiante ingresa a una sala para registrarse en sus cursos universitarios. Mientras se acerca a la mesa de registro, el estudiante aparenta que sus libros y papeles se caen casualmente, cuando en realidad los ha soltado adrede. Luego de eso, los recoge y se registra exitosamente en sus cursos. A un grupo de sujetos que observa al estudiante se le indica que la meta de este es completar un grado en química, mientras que a otro grupo se le indica que su meta es obtener un grado en música. Dependiendo de la información brindada, las personas que observan el experimento tienden a crear memorias consistentes con esos esquemas. Así, por ejemplo, los sujetos que creen que la meta del estudiante es completar un grado en música, afirman que aquel casi pierde sus materiales de música, pero que finalmente logró matricularse en clases de música. ${ }^{30}$

Como afirman Cantor y Mischel, los "esquemas sobre personas" nos ayudan a categorizar, recordar y derivar inferencias acerca de las personas que observamos. Empero, como demuestran los experimentos citados, estos esquemas pueden producir conclusiones erradas. ${ }^{31}$

La importancia de los "esquemas sobre personas".es que pueden ser aplicados en varios niveles, desde un individuo hasta grupos de personas. Ciertamente, la tendencia de ver a las personas de acuerdo a esquemas sobre sus características y sus metas es una manifestación de una tendencia a atribuir o entender los comportamientos sobre la base de la disposición más que de la situación de la persona.

\section{Esquemas sobre Roles (Role Schemas)}

Los"esquemas sobre roles" nos ayudan a organizar el conjunto de comportamientos esperados de una persona en una posición social particular. ${ }^{32}$ Estos esquemas se aplican a roles que son logrados, adscritos o adquiridos al nacer. ${ }^{33}$ Como los "esquemas sobre personas" y los "esquemas individuales", los "esquemas sobre roles" permiten dar sentido a las características y los comportamientos de otras personas, así como a predecir sus reacciones. En buena cuenta, los "esquemas sobre roles" nos ayudan a saber qué esperar y qué no de los demás. ${ }^{34}$ Así, por ejemplo, nosotros no nos sorprenderíamos si en una cita con el médico este nos pida que digamos

28 CHEN, Ronald y Jon HANSON, Op. Cit., p. 1135

29 Ibídem.

30 ZADNY, Jerry y B. GERARD, Harold, Attributed Intentions and Information Selectivity. En: Journal of Experimental Social Psychology, No. 10, 1974, pp. 34 y ss.

31 CANTOR, Nancy y MISCHEL, Walter, Prototypes in Person Perception. En: Advances in Experimental Social Psychology Vol. 12, Editor: BERKOWITZ, Leonard, Academic Press, New York, 1979, pp. 3-6.

32 FISKE, Susan y TAYLOR E., Shelley, Social Cognition, McGraw Hill Higher Education, New Jersey, 1991, p. 119.

33 lbídem.

34 CHEN, Ronald y HANSON, Jon, Op. Cit., p. 1137. 
"ahhhhhhhhh" o que tosamos, pero esto nos sorprendería terriblemente si es que quien nos pide que hagamos esto es nuestro contador. De otro lado, jamás esperaríamos que en una cita con el médico, este nos pida nuestra declaración jurada de impuesto a la renta del ejercicio anterior, cosa que sería normal en una cita con nuestro contador. ${ }^{35}$

Los "esquemas sobre roles" y los "esquemas sobre personas" son en realidad parte de lo que configuran los estereotipos, que nos sirven para organizar nuestras expectativas acerca de otras personas que encajan en ciertas categorías sociales. ${ }^{36}$ Justamente es por eso que dentro de nuestro estereotipo sobre un árbitro de fútbol, pensemos, entre otras cosas, en un hombre y no una mujer.

Como explican Hanson y Chen, los "esquemas sobre roles" y los"esquemas sobre personas" pueden aplicarse tanto a seres humanos como a entidades e instituciones que tendemos a "personalizar" (desde corporaciones hasta naciones, desde entidades estatales hasta grupos de interés). ${ }^{37}$

\section{Esquemas sobre Eventos (Event Schemas)}

Finalmente, los "esquemas eventos", también denominados scripts, nos ayudan a comprender los distintos pasos o secuencias involucradas en un proceso determinado. ${ }^{38}$

Pensemos en el ejemplo del dentista: a muchos de nosotros nos sorprendería gratamen- te que aquel nos reciba en la sala de espera y nos invite personalmente a pasar, pero nos parecería extraño que pretenda revisar nuestra dentadura en la sala de espera y no en el consultorio. ${ }^{39}$

Pensemos ahora en el ejemplo de los restaurantes: los individuos estamos generalmente familiarizadas con los scripts de los restaurantes, que contienen información que nos ayuda a entender y a predecir la secuencia de los eventos. ${ }^{40}$ Cuando vamos a un restaurante sabemos, en la gran mayoría de los casos, que una vez sentados, el mozo vendrá y nos dará un menú. Luego de revisarlo, ordenaremos lo que nos apetezca y podamos pagar. Posteriormente, el mozo nos traerá la comida y luego la cuenta. Tan pronto la paguemos podremos sentirnos libres de abandonar el local. ${ }^{41}$

Los scripts nos permiten entender e interpretar mejor las cosas que vemos y las que no vemos. ${ }^{42}$ Regresando al ejemplo del restaurante, si vemos a una persona pagando la cuenta y retirándose del restaurante, nuestro script sobre restaurantes nos indicará que previamente se sentó, revisó el menú, llamó al mozo y ordenó algo. Así, por los scripts que manejamos, inferimos hechos que no hemos visto, deducimos hechos que si bien no nos constan, seguramente se produjeron. ${ }^{43}$

La aplicación de scripts suele ser, sin embargo, automática, no razonada. Esto puede generar que ignoremos lo que no vemos aunque sea real 0 , lo que es peor, que ignoremos to que debemos ver. ${ }^{44}$

\footnotetext{
35 CHEN, Ronald y HANSON, Jon, Op. Cit., p. 1137.

36 FISKE, Susan y E. TAYLOR, Shelley, Op. Cit., p. 119.

37 CHEN, Ronald y HANSON, Jon, Op. Cit., p. 1137.

38 Ibídem.
}

39 Ejemplo basado en el trabajo de CHEN, Ronald y HANSON, Jon, Op. Cit., p. 1138.

40 CHEN, Ronald y HANSON, Jon, Op. Cit., p. 1138.

41 Ibidem, p. 1138.

42 Ibídem, p. 1139.

43 Ibidem.

44 Ibídem. 


\section{Paradojas}

El empleo de categorías y esquemas conduce a resultados paradójicos, originados quizás por nuestras limitaciones cognitivas. ${ }^{45} \mathrm{En}$ efecto, las categorías y los esquemas han sido creados para representar la realidad que nos rodea en condiciones tales que los componentes de esa realidad resulten procesables y administrables por nosotros. La experiencia, sin embargo, demuestra que generalmente empleamos categorías y esquemas para crear una meta realidad, que resulta de la tendencia que tenemos de explicar los nuevos fenómenos en función de categorías y esquemas pre-existentes; tendencia que encuentra su explicación en nuestras propias limitaciones cognitivas y en el deseo de obtener beneficios al menor costo posible. Así, en lugar de analizar si los nuevos fenómenos responden realmente a categorías y esquemas pre-existentes $y$, de ser el caso, de desarrollar nuevas categorías y esquemas para representar correctamente la realidad, tratamos de explicar dichos fenómenos en función de categorías y esquemas ya desarrollados, pues ello resulta "menos costoso". En otras palabras, en lugar de tratar de desarrollar categorías y esquemas apropiados para nuevos hechos, forzamos el ingreso de estos últimos en categorías ya desarrolladas y, en consecuencia, explicamos estos nuevos sucesos en función de tales categorías y esquemas, aun cuando no tengan correspondencia. De este modo, las categorías y esquemas nublan nuestra vista, adormecen nuestros sentidos y refuerzan la tradición en su más nocivo sentido.

En nuestra opinión, la categorización del dominio fiduciario como derecho real es un lamentable resultado de la fuerza viva de la vocación por el mínimo esfuerzo, que sujeta a la profesión legal a cánones de otros siglos y sociedades.

\section{CONCEPTOS LEGALES}

En el Derecho, como en cualquier otra disciplina, los operadores usamos categorías y esquemas. Las primeras nos permiten procesar la información legalmente relevante y los segundos resolver la duda o controversia existente sobre las consecuencias legales de los hechos dados.

Evidentemente, las categorías y los esquemas no tienen por qué ser inmutables. Los operadores legales podemos ponernos de acuerdo tanto para modificar las formas en las que clasificamos los eventos legalmente relevantes como para modificar las apreciaciones cognitivas que desarrollamos sobre tales eventos. Si en los hechos no lo hacemos es porque seguramente los costos del proceso superan a los beneficios.

Las categorías y los esquemas tampoco tienen por qué determinar la solución legal en función de criterios lógicos formales. Si bien los operadores legales necesitamos categorizar los problemas legalmente relevantes y desarrollar un conjunto de conocimientos sobre ellos, no hay razón alguna para que las soluciones reclamadas por la comunidad sean creadas sobre la base de inferencias lógicas formales ("el contrato es vinculante; por tanto, ante su incumplimiento la parte afectada debe poder solicitar la ejecución forzada y/o el pago de una indemnización"), en tanto que tales inferencias pueden conducir a resultados ineficientes o incluso inmorales.

La significativa trascendencia del criterio lógico formal en la solución de los problemas con relevancia legal, privativa del sistema romano-germánico ${ }^{46}$, es el resultado irónico del predominio de las categorías y los esquemas desarrollados por la comunidad científica europea del siglo XVII; en especial, es el resultado de una aplicación excesiva de los"esquemas sobre eventos"que definieron el método científico sobre la base del modelo de la geometría. Veamos cómo ocurrió esto.

45 Ibidem.

46 En el Common Law la solución de los problemas con relevancia legal se basa fundamentalmente en policy considerations, esto es, en razones de política social que hacen depender la decisión de las cortes de los incentivos y desincentivos que una regla legal generaría en los diversos actores de la sociedad. 
A raíz del redescubrimiento de las Institutas de Justiniano ${ }^{47}$, el Derecho Romano, fundamentalmente casuístico, recobró cierta vigencia en Europa continental gracias a la poderosa influencia gremial que en ese entonces ejerció la Universidad de Bologna. En el siglo XVII, sin embargo, el conocimiento casuístico era sinónimo de conocimiento asistemático y, por consiguiente, de conocimiento no científico. La comunidad cientifica de entonces veía en la geometría el modelo que la ciencia en general debía seguir ${ }^{48}$, en tanto que, al posibilitar la obtención de teoremas mediante inferencias derivadas de axiomas, el método geométrico ofrecía la recompensa de una verdad atemporal libre de las vaguedades de la labor empírica. Por tal razón, Descartes y otros pensadores de la época consideraban que la geometría era la ciencia que debía inspirar a todas las demás disciplinas en su pretensión de convertirse en ciencias. ${ }^{49}$

El sabio Gottfried Wilhem Leibniz (1646-1716) -abogado, físico, filósofo, geómetra, ingeniero, político-buscó desde temprana edad revitalizar el poder del Derecho a través de una aproximación científica que prometiese un conocimiento verdadero y cierto de la ley natural, de modo tal que nadie pudiese dudar de su existencia y autoridad. ${ }^{50}$ Para ello se basó en el principio de la razón suficiente e impulsó un cambio trascendental en el foco de atención del estudio del Derecho: en lugar de conocer lo que la ley establecía, había que conocer las razones, bases y justificaciones de eso que la ley establecía. ${ }^{51}$

Partiendo de la idea final de que el Derecho debía basarse en el ideal de justicia según la voluntad de Dios, Leibniz se embarcó en la elaboración de un código cientifico, aplicando para ello el método de la geometría. ${ }^{52} \mathrm{~A}$ diferencia de los códigos antiguos (Hammurabi por ejemplo), que eran una colección de leyes válidas, el código científico era una colección de normas válidas sistemáticamente ordenadas, que podían ser lógicamente deducidas de principios fundamentales. ${ }^{53}$

El Codex Leopoldus, la obra científica de Leibniz, estaba organizado sobre la base de axiomas y teoremas. De este modo, así como los más diversos teoremas geométricos podían ser explicados a partir de unos cuantos axiomas elementales, las reglas legales contenidas en dicho código podian ser lógicamente derivadas de unos pocos principios de justicia. El objetivo confeso de Leibniz era reducir todo el Derecho Romano a sus principios generales (axiomas) para derivar de tales principios, mediante procedimientos lógicos, todas las reglas legales que la comunidad necesitase (teoremas), de modo tal que cualquier persona común que conociera los axiomas (que no eran numerosos) pudiese resolver el más intrincado problema legal a través de un sencillo proceso deductivo. ${ }^{54}$

47 Al rededor del año 1080, en una libreria italiana se descubrió una copia de las Institutas de Justiniano. El evento conmocionó al incipiente mundo académico de entonces, que recibió la noticia como si se tratase del hallazgo de una copia perdida del antiguo testamento. Ver: BERMAN, Harold 1., Law and Revolution, Harvard University Press, Cambridge, 1983, p. 123.

48 BERKOWITZ, Roger, The Gift of Science. Leibniz and the Modern Legal Tradition, Harvard University Press, Cambridge London, 2005, p. 17.

49 Luc. Cit.

50 BERKOWITZ, Roger, Op. Cit., p. 7.

51 Luc. Cit.

52 Es muy probable que la idea de Leibniz de combinar geometria y leyes tuviera su origen en los trabajos de Erhard Wiegel, su maestro en teoría de la probabilidad, quien, además de ser un erudito en aritmética, tenía profundos conocimientos sobre jurisprudencia. Para mayor referencia: HACKING, lan, El Surgimiento de la Probabilidad, Traducido por ÁLVAREZ, José A., Editorial Gedisa, Barcelona, 2005, p. 109 y ss.

53 BERKOWITZ, Roger, Op. Cit., pp. 20 y 21.

54 BERKOWITZ, Roger, Op. Cit., p. 26. 
El Emperador Leopoldo I rechazó el pedido de Leibniz de convertir al Codex Leopoldus en la ley suprema del Imperio Austro-Húngaro. No obstante, con esa obra Leibniz marcó para siempre el destino de una buena parte de la humanidad, pues su método científico no solo inspiró a la Escuela Histórica fundada por Friedrich Carl von Savigny sino que se vio finalmente reflejado en la arquitectura del $\mathrm{BGB}^{55}$ y en la escuela de la jurisprudencia de conceptos. ${ }^{56}$

El resultado último de la labor de Leibniz fue ciertamente irónico. El sabio quiso acercar la ciencia a la justicia para que esta última se fortaleciera; no obstante, la concepción del Derecho que heredamos de aquel, esa que opera con axiomas y teoremas conectados por procesos lógicos formales, privilegia la forma sobre la sustancia, la coherencia deductiva sobre lo económicamente eficiente o moralmente exigible; en suma, el "conocimiento" sobre la justicia. ${ }^{57} \mathrm{De}$ no haber existido un script geométrico sobre el proceso científico, quizás hoy tendríamos un sistema legal mucho más justo y eficiente.

En términos psicológicos, la concepción científica del Derecho ha creado una supra-realidad en la que los problemas con relevancia legal son explicados y tratados, no en función de costos, beneficios, incentivos, desincentivos, valores, etc., sino en función de conceptos legales. Asi, por ejemplo, no interesa si, en ejercicio de su libertad individual, dos personas informadas adoptan un acuerdo que genera beneficios recíprocos $y$, por tanto, maximiza su estado de bienestar y el de la sociedad. Si ese acuerdo no tiene "efectos obligacionales" (no crea, modifica o extingue obligaciones $)^{58}$, entonces ese acuerdo no constituye un "contrato" 59 y, por consiguiente, esas personas no tienen la

55 BERKOWITZ, Roger, Op. Cit., p. 137 y ss.

56 Es interesante notar que el Common Law también sucumbió a los encantos de la escuela científica. En efecto, en Inglaterra William Blackstone mostró en su tratado que el Common Law tenía orden interno y fundamentos racionales que podían explicar, desde una perspectiva lógica, todas las soluciones legales. Ver: SUTHERLAND, Arthur, The Law at Harvard, Harvard University Press, Cambridge, 1967, p. 24 y ss. En los Estados Unidos de América, por su parte, Christopher Columbus Langdell implantó el método geométrico de Leibniz, construyendo un sistema teórico que explicaba, desde una perspectiva lógica, todas las decisiones judiciales a partir de unos cuantos principios fundamentales. Ver: KRONMAN, Anthony, The Lost Lawyer, The Belknap Press of Harvard University Press, Cambridge - London, 1993, p. 171. El acercamiento entre Derecho y ciencia terminó, por lo menos en los Estados Unidos de América, de manera abrupta con el feroz ataque emprendido primero por el Juez de la Corte Suprema de los Estados Unidos de América Oliver Wendel Holmes, y luego por el profesor Jerome Frank y todos los demás proponentes del Legal Realism.

57 Como observan Kevin Gray y Susan Francis Gray, "[t]he essence off an axiomatic structure is, of course, that 'axioms' (or 'fixed postulates') enjoy a fundamental and autonomous status within the scheme. An axiomatic proposition is a raw datum whose validity is assumed, never proved. One can no more seek to ho behind an axiom than one may question why, in a game of chess, the knight or the bishop moves in the peculiar ways these pieces do. It is irrelevant that the axiom in question is morally neutral or indifferent and that no evil of any kind would ensue from a denial of its validity. Axioms are simply immune from rational challenge. To knock at an axiom is to want to play a different game; and to alter an axiom is already to have begun a different game". Ver: The Rhetoric of Reality. En: Rationalizing Property, Equity and Trusts. Essays in Honour of Edward Burn. Editor: GETZLER, Josua, Lexis Nexis, 2003, pp. 208 y 209.

58 Las partes pueden adoptar reglas que no contengan pretensiones ni deberes. Por tal razón, los acuerdos privados pueden no tener efectos obligaciones. Contrariamente a lo que sostienen muchos autores locales, el Código Civil peruano contempla una serie de supuestos en los cuales los contratos no generan efectos obligacionales (p.e. promesa de hecho ajeno y cesión de posición contractual). Evidentemente, eso significa que las normas del referido código son contradictorias, pues algunos de sus artículos sugieren una lectura "obligacional" en materia de eficacia contractual. El problema, sin embargo, no se soluciona homogenizando las referidas normas en un sentido restrictivo, esto es, en un sentido que limite los alcances del contrato al plano "obligacional", pues tal cosa sería inmoral e ineficiente y, por tal razón, inconstitucional a la luz de lo dispuesto por el artículo 3 de nuestra Constitución Política.

59 En tal sentido: ARIAS SCHEREIBER, Max, Exégesis del Código Civil Peruano de 1984, Tomo l, San Jerónimo, Lima, 1988 , pp. 76, 77, 97, 98, 154 y 155; DE LA PUENTE Y LAVALLE, Manuel, El Contrato en General, Pontificia Universidad Católica del Perú - Fondo Editorial, Primera Parte, Tomo I, Lima, p. 96 y ss. 
protección legal que el Código Civil dispensa a quienes sí han celebrado un acuerdo con "efectos obligacionales" ("contrato"). Como es obvio, no existe razón moral o económica alguna que justifique la decisión de no otorgar reconocimiento legal a acuerdos libremente adoptados y mutuamente beneficiosos. Por lo tanto, en lugar de ayudarnos a procesar los datos de la realidad para reconocer aquellos casos en los cuales los acuerdos requieren, a la luz de políticas públicas de bienestar, reconocimiento y protección legal, el concepto legal de contrato castra la vida social, menospreciando nuestra dimensión moral e impidiendo que los recursos escasos sean reasignado a sus usos alternativos más beneficiosos. De esta forma, el Derecho crea un mundo en el que los criterios económicos y morales son opacados por la naturaleza jurídica de sus habitantes, como si la ley fuera un fin y no un medio para conseguir paz y bienestar.

\section{NOCIÓN DE DERECHO}

¿Qué es un derecho? Revisemos brevemente algunas nociones fundacionales de la cultura occidental, a luz del pensamiento de algunos de sus principales exponentes.

Thomas Hobbes consideraba que otorgar un derecho a un individuo sobre algo significaba dar a ese individuo la libertad para gozar libremente de ese algo y alcanzar sus propios fines. ${ }^{60}$

Immanuel Kant sostenía que cuando un individuo tenía la permisión moral o la libertad de hacer algo sin que otros tuvieran un deber correlativo de respeto (piénsese en el derecho del prisionero de guerra a escapar), ese individuo tenía un "derecho imperfecto". En cambio, cuando un individuo tenía la capacidad de obligar a otros a respetar su libertad, ese individuo tenía un "derecho perfecto". Si el deber correlativo era exigible a través de la coerción, entonces el individuo tenía un "derecho legal"; si no lo era, entonces el individuo tenía un "derecho moral".61

John Stuart Mill consideraba que un individuo tenía un derecho cuando aquel podía válidamente acudir a la sociedad a fin de reclamar protección para una "posesión", esto es, para algo que le generaba utilidad. ${ }^{62}$

John Austin sostenía que un individuo era titular de un derecho cuando ese individuo resultaba beneficiado sea de la imposición de un deber a otro sea de la exoneración de un deber que en principio le hubiese correspondido cumplir. ${ }^{63}$

Como se podrá advertir, en el terreno filosófico y político el concepto de derecho ha sido cimentado sobre la base de dos ideas centrales: libertad y beneficio. De este modo, consideramos que alguien tiene un derecho cuando ese alguien recibe un beneficio con libertad, esto es, cuando ese alguien es protegido sin ser forzado a reclamar la protección conferida. ${ }^{64}$

Desde una perspectiva legal, nuestros esquemas y categorías no difieren de los que la teoria filosófica y política emplea para definir a los derechos. En efecto, todas las posiciones en torno al derecho subjetivo (facultas agendi, interés protegido, protección del interés, etc.) coinciden en que el referido derecho supone dos cosas: libertad de actuar e interés propio. ${ }^{65}$

60 SORELL, Tom, Hobbes, Routledge, London - New York, 1986, p. 112.

61 ROSEN, Allen, Kant's Theory of Justice, Cornell University Press, Ithaca - London, 1993, p. 82 y ss.

62 STUART MILL, John, Utilitarianism. En: The English Philosophers, Editor: A. BURTT, Edwin, Random House, Inc., New York, 1939, p. 938.

63 AUSTIN, John, Lectures on Jurisprudence, or the Philosophy of Positive Law, John Murria, London, 1885, p. 54 y ss.

64 En este sentido: HAUSMAN, Daniel M. y MCPHERSON, Michael S., El Análisis Económico del Derecho y la Filosofía Moral, Traducido por GROBET VALLARTA, Mauricio Luis, Fondo de Cultura Económica, México, 2007, pp. 201-202.

65 Una exposición de las diversas teorías sobre el derecho subjetivo se encuentra en: ESCOBAR, Freddy, Teoría General del Derecho Civil. Cinco Ensayos, ARA Editores, Lima, 2002, p. 125 y ss. 
En este sentido, una situación legal es calificada como situación de derecho solo si el sujeto involucrado en aquella tiene la facultad de actuar para satisfacer un interés propio. Si el sujeto en cuestión no tiene la facultad de actuar, sino la necesidad de hacerlo para satisfacer un interés ajeno, entonces la situación legal es calificada como situación de deber. ${ }^{66}$ De estas ideas sencillas se derivan diversas consecuencias legales trascendentales. ${ }^{67} \mathrm{He}$ aquí las más notorias.

Si el derecho comprende una facultad de actuar para satisfacer un interés propio, entonces no castigamos a quien no ejerce sus derechos y de ese modo no satisfaga sus propios intereses, salvo que de manera muy excepcional consideremos que tal situación es nociva (abuso del derecho) en términos de bienestar agregado. Asimismo, permitimos que los sujetos renuncien y transfieran libremente sus derechos, pues generalmente tal cosa es indiferente (no perjudica) para aquellos que han de respetar esos derechos. Y cuando no lo es, aceptamos que se elimine y que se restrinja la posibilidad de que los sujetos transfieran libremente sus derechos. ${ }^{68}$

Por su parte, si el deber comprende la necesidad de actuar para satisfacer un interés ajeno, entonces castigamos de modos muy diversos a quien se desentiende de tal necesidad e impide de esa manera la satisfacción de un interés ajeno. ${ }^{69}$ Por otro lado, impedimos que los sujetos renuncien o transfieran libremente sus deberes, pues generalmente tal cosa no es indiferente para aquellos que han de beneficiarse con el cumplimiento de los mismos.

A la luz de estos esquemas y categorías, ¿podemos afirmar que el dominio fiduciario pertenece al mundo de los derechos?

\section{v. FUNCIONES DEL FIDEICOMISO}

El fideicomiso latinoamericano tiene una historia relativamente larga. El primer intento de instaurar esta figura en un ordenamiento latinoamericano se remonta a los primeros años del siglo pasado, época en que las grandes empresas estadounidenses solicitaron a los gobiernos latinoamericanos-deseosos de recibir inversión extranjera- la adopción de una figura legal que les permitiese organizarse a semejanza del Trust..$^{70} \mathrm{Y}$ es que si bien el Trust nació como un derecho de propiedad protegido por la Equity ${ }^{71}$ y por tanto paralelo al derecho de propiedad protegido por el Common Law, a inicios del siglo pasado no existían más las viejas limitaciones

66 Una exposición de las diversas teorías sobre el deber jurídico se encuentra en: ESCOBAR, Freddy, Teoría General (...), pp. 188 y ss.

67 Los derechos y los deberes no agotan ciertamente la totalidad de situaciones legales de los individuos. En efecto, los individuos usualmente tienen facultades, privilegios, poderes, inmunidades, sujeciones, responsabilidades y otras situaciones que no poseen la estructura de lo que denominamos derecho o deber. Para una exposición detallada de las diversas situaciones legales que se presentan en la realidad: HOFELD, Wesley, Some Fundamental Legal Conceptions as Applied in Judicial Reasoning. En: Yale Law Journal, Número 23, 1913, p. 30 y ss.; SPERDUTI, Giuseppe, Contributo alla Teoria delle Situazioni Giuridiche Soggettive, Dott. A. Giuffre Editore, Milano, 1944, p. 4. y ss.

68 La eliminación y la restricción de esa posibilidad están sustentadas tanto en razones morales como en razones económicas. Así, por ejemplo, impedimos la venta órganos para evitar tanto externalidades psicológicas ("moralismos") como económicas (si la comercialización de órganos estuviese permitida, podrían generarse mayores incentivos para el robo de órganos al tratarse de bienes que resultarían más fácilmente comercializables en el mercado).

69 Los castigos que aplicamos incluyen medidas tan diversas como la expropiación de bienes y la pérdida de la libertad personal.

70 La secuencia de estos hechos se encuentra descrita en: ESCOBAR, Freddy, Traditions, Transplants and Inefficiencies: The Case of the Peruvian Fideicomiso. En: Vniversitas. Revista de la Facultad de Ciencias Juridicas de la Pontificia Universidad Javierana, Número 111, 2006, p. 176.

71 Equity es el término que se utiliza en el Common Law para describir al conjunto de reglas y principios que las cortes aplican cuando un caso no puede ser resuelto por los precedentes del Common Law, ni por las normas escritas del Statutory Law. Tales reglas y principios están orientados hacia la obtención del fairness. 
legales que habían impulsado el desarrollo del Trust en el campo de los derechos reales. Por tal razón, la comunidad norteamericana comenzó a emplear el Trust con otros propósitos: realización de transferencias gratuitas y creación de organizaciones empresariales. ${ }^{72}$

Como relata Sitkoff ${ }^{3}$, a inicios del siglo pasado, en los Estados Unidos de América el Trust dominaba a la Corporation como forma organizacional en las industrias más relevantes ${ }^{74}$, al punto que el problema de los monopolios y de las prácticas anticompetitivas fue básicamente generado por grandes empresas organizadas bajo la forma de Trusts. Por tal razón las leyes antimonopolio recibieron el nombre de leyes Antitrust y no de leyes Anticorporation. Con el tiempo la lucha entre la Corporation y el Trust ha generado resultados divididos. Así mientras la Corporation domina ciertos sectores como los industriales y los de servicios, el Trust domina otros sectores como el de las finanzas estructuradas, las titulizaciones y los fondos mutuos. ${ }^{75}$

Todos los modelos de fideicomiso latinoamericano han querido basarse en el Trust. ${ }^{76} \mathrm{El}$ Trust, sin embargo, es un instituto complejo, en constante evolución, con usos múltiples. Así, el Trust puede ser empleado como instrumento sucesorio, como mecanismo de emisión de valores, como remedio judicial, etc. Por tal razón, la regulación del Trust es sumamente diversa y no siempre homogénea. De este modo, por ejemplo, mientras que el Uniform Trust Code "(...) is directed primarily at trusts that arise in an estate planning or other donative context (...)", el Delaware Business Trust Act " $(.$.$) is directed at bu-$ siness trusts as alternative forms of entities $(\ldots)^{\prime 77}$ Por consiguiente, el legislador latinoamericano ha de tener extremo cuidado si desea regular el fideicomiso a la luz del Trust. ${ }^{78}$

En el Perú, el Fideicomiso no está regulado en el Código Civil, sino en la Ley de Mercado de Valores y en la Ley General del Sistema Financiero y del Sistema de Seguros y Orgánica de la Superintendencia de Banca y Seguros. Estas leyes no permiten constituir fideicomisos con la libertad que garantiza los artículos 1353 y 1354 del referido código. No obstante ello, hay que reconocer que los usos que las leyes en cuestión permiten dar al fideicomiso son relativamente diversos. Así, podemos constituir, entre otros, fideicomisos con fines de garantía, administración y/o titulización.

72 Como señala LANGBEIN, John, "[t]he feudal restrictions on the transfer of real property that gave rise to the conveyancing-type trust disappeared piecemeal from the late seventeenth to the early twentieth centuries. Although feudal land law no longer needs evading, the trust has endured because it has changed function. The trust has ceased to be a conveyancing device for holding freehold land and has become instead a management device for holding financial assets. The management trust is a response to the radical change away from family real estate as the dominant form of wealth". Ver: The Contractarian Basis of the Law of Trusts. En: Yale Law Journal, Número 105, 1995, p. 637.

73 SITKOFF, Robert, Trust as "Uncorporation": A Research Agenda. En: University of Illinois Law Review, Vol. 2005, p. 32.

74 John Rockefeller organizó la Standard Oil Company no como Corporation sino como Trust.

75 En tal sentido: SITKOFF, Robert, Op. Cit., p. 44; SCHWARCZ, Steven, Commercial Trusts as Business Organizations: An Invitation to Comparatists. En: Duke Journal of Comparative \& International Law, Vol. XII, 2003, p. 321.

76 En palabras de MOISSET DE ESPANÉS, "[e]l fideicomiso que hoy se incorpora a nuestra normativa es una figura inspirada en el trust anglosajón". Ver: El Fideicomiso (Ley 24.441). En: Revista del Notariado, Número Extraordinario, 1995, p. 2. La historia del fideicomiso en Latinoamérica se encuentra detallada en: ESCOBAR, Freddy, Traditions(...), pp. 176 a 186.

77 La regulación del Trust es difusa y compleja. Así, al Restatement of Trusts, que recoge los principios del Common Law en la materia, se le suman, como normas positivas, entre muchas otras, el Uniform Trust Code, el Uniform Commercial Code, el Prudent Investor Rule y el Uniform Trustees' Powers Act.

78 Un magnífico ejemplo de correcto entendimiento de la función organizacional del fideicomiso se encuentra en la regulación ecuatoriana. En efecto, la Ley 107 de 1998 (Ley del Mercado de Valores) establece que el fideicomiso es un patrimonio autónomo y que el fiduciario es el representante legal de dicho patrimonio. 
Desde una perspectiva económica, ¿qué tienen en común un fidecomiso de garantía sobre un terreno y un fideicomiso de administración de un portafolio de inversiones? ¿Qué tienen en común un fideicomiso de titulización de flujos futuros y un fideicomiso cultural? El factor que hermana a todos estos fideicomisos es el de la creación de un patrimonio autónomo, esto es, el de la creación de un conjunto de bienes que no forman parte de los patrimonios del fideicomitente, del fideicomisario y del fiduciario. Al margen de este factor, ¿qué puede hermanar a todos estos fideicomisos?

Si pensamos en categorías legales, probablemente coloquemos al fideicomiso de garantía en la caja de los "fenómenos reales" y al fideicomiso de titulización en la caja de los "fenómenos organizacionales", en tanto que en el primer caso el fiduciario solo tiene que vender el terreno en caso de incumplimiento, mientras que en el segundo caso el fiduciario tiene que encargarse de la emisión de bonos, del cobro de los flujos, del pago del capital e intereses de los bonos, de la defensa del patrimonio, etc. ${ }^{79}$ ¿No deberíamos tomar en cuenta estas distintas funciones de los fideicomisos en cuestión para regularlos y explicarlos de manera diversa? ¿O el hecho que en ambos casos existan patrimonios autónomos basta para considerar que esos fideicomisos pertenecen al mundo de los "fenómenos reales"?

Si el Trust es considerado tanto un "fenómeno real" (property in Equity) como un "fenómeno organizacional", ¿no deberíamos esquematizary categorizar al fideicomiso bancario y financiero como un "fenómeno organizacional"?

\section{DOMINIO FIDUCIARIO EN EL SISTEMA LEGAL PERUANO}

¿Qué es lo que tienen en común el fiduciario (en su condición de titular del dominio fiduciario) y el propietario? La posibilidad de realizar determinados actos (p.e. vender o arrendar) sobre ciertos bienes. ¿Qué es lo que diferencia al fiduciario (en su condición de titular del dominio fiduciario) del propietario? Mientras el propietario puede o no realizar los actos en cuestión, satisfaciendo en cualquier caso un interés propio; el fiduciario tiene que realizar esos actos, satisfaciendo en cualquier caso un interés ajeno (el del fideicomisario).

¿Qué es lo que tienen en común el fiduciario y el administrador (gerente o director) de una sociedad anónima? La posibilidad de realizar determinados actos sobre ciertos bienes. ¿Qué es lo que diferencia al fiduciario del administrador de una sociedad anónima? Mientras el administrador es parte de la estructura organizativa de la persona jurídica. propietaria de los bienes, el fiduciario no es parte de una estructura como esa.

El fiduciario no ejerce el dominio fiduciario para satisfacer sus propios intereses sino para satisfacer los intereses del fideicomitente $y$ del fideicomisario. Por otro lado, el fiduciario no tiene libertad para decidir si ejerce. o no el referido dominio, sino más bien la necesidad de hacerlo, bajo pena de perder su condición de tal y de pagar una indemnización por los daños que ocasione su omisión. Por tal razón, es obvio que el fiduciario no tiene un derecho.

Sin perjuicio de lo indicado, es necesario prestar atención a un dato normativo absolutamente revelante, contenido tanto en el artículo 241 de la Ley General del Sistema Financiero y del Sistema de Seguros y Orgánica de la Superintendencia de Banca y Seguros, como en el artículo 310 de la Ley del Mercado de Valores. ¿Qué establecen esos artículos? Veamos.

El primero establece que "[e]/ patrimonio fideicometido es distinto al patrimonio del fiduciario, del fideicomisario, o del fideicomitente, y en su caso del destinatario de los bienes remanentes". El segundo, por su parte, establece que "[e]/acto constitutivo, una vez cumplida la formalidad esta-

79 En palabras de SCHWARCZ, "securitization transactions exemplify the seemingly interchangeable use of trusts and corporations as forms of business organizations". Ver: Op. Cit., p. 333. 
blecida, genera un patrimonio autónomo, distinto al patrimonio propio de la sociedad titulizadora, del fideicomitente, del fideicomisario y de la persona designada como destinatario de los activos remanentes del fideicomiso".

Tomando en consideración este dato positivo, ¿podemos seriamente considerar que el fiduciario tiene un derecho real sobre bienes que, según el ordenamiento vigente, no forman parte de su patrimonio ni del patrimonio de las otras partes intervinientes? ${ }^{80}$

$\mathrm{Si}$, como es evidente, el dominio fiduciario, no es asimilable al derecho, ¿cómo categorizarlo? ¿Cómo esquematizarlo?

Lo único que diferencia al fiduciario del administrador es la existencia de una persona jurídica. ${ }^{81}$ En efecto, ambos gestionan intereses de otros, ambos se obligan a realizar ciertas actividades en beneficio de otros y ambos responden con sus patrimonios en caso incumplan con cuidar los intereses de otros. Ahora bien, ¿la inexistencia de una persona juridica en la estructura del fideicomiso determina la imposibilidad de asimilar al fiduciario con el administrador? En absoluto, pues la figura del patrimonio autónomo cumple en el caso del fiduciario la misma función que la figura de la persona jurídica cumple en el caso del administrador. En efecto, al igual que la persona jurídica, el patrimonio autónomo posee subjetividad jurídica, es decir, capacidad para contratar, adquirir derechos, obligaciones y demás situaciones legales, demandar y ser demandado, etc. Esto determina que, al igual que la persona jurídica en el caso del administrador, el patrimonio autónomo instaure un velo entre el fiduciario y los bienes sobre los cuales aquel ejerce sus funciones. Sin ese velo, el fiduciario sería titular de los bienes, aunque bajo un esquema fiduciario, no fideicomisario. ${ }^{82}$

La figura del patrimonio autónomo resuelve cualquier inconsistencia teórica que se encuentre entre el rol del fiduciario y la inexistencia de una persona jurídica. ${ }^{83} \mathrm{Al}$ ser un ente provisto con subjetividad jurídica ${ }^{84}$, el patrimonio autónomo requiere de un administrador y de un

80 Para un mayor desarrollo sobre la inconsistencia entre la noción de patrimonio y la de dominio fiduciario como derecho real: ESCOBAR, Freddy, Traditions (...), p. 194 y ss.

81 Evidentemente, esto no implica que aboguemos por una asimilación total entre el tratamiento legal del fiduciario y el tratamiento legal del administrador de una sociedad de capitales. En efecto, el fideicomiso y la sociedad de capitales son fenómenos organizacionales con caracteristicas y fines distintos. Así, mientras el fideicomiso sirve para desarrollar propósitos especiales, perfectamente definidos por el fideicomitente; la sociedad de capitales sirve para realizar actividades empresariales, genéricamente definidas por los accionistas. Por tal razón, mientras el margen de acción del fiduciario es relativamente reducido, el margen de acción del administrador es relativamente amplio. Estas diferencias fácticas determinan que ambos fenómenos organizacionales presenten caracteristicas propias en torno a los problemas de los costos de agencia, de los efectos reputacionales, etc. Por tal razón, el régimen legal de responsabilidad aplicable al fiduciario y al administrador no puede ser idéntico.

82 El esquema fiduciario (negocio atípico) es muy distinto del esquema fideicomisario (negocio típico), pues, a diferencia de este último, aquel genera efectos que exceden los que normalmente producen los esquemas de los cuales se nutre (p.e. venta con fines de garantía). Ver: ESCOBAR, Freddy, Contribución al estudio de los negocios fiduciarios. En: Negocio Juridico y Responsabilidad Civil. Estudios en Memoria del Profesor Lizardo Taboada Córdoba, Editores: ESCOBAR, Freddy; MORALES, Rómulo; LEÓN, Leysser y PALACIOS, Eric, Editora Grijley, Lima, p. 463 y ss.

83 Comentando la solución al problema indicado, PENNER, Stephen M. afirma lo siguiente: "The answer to the latter difficulty has been found, in some jurisdictions, in the idea of a separate trust 'patrimony' or 'estate'. The patrimony of the trust, on this analysis, is distinct from the private patrimony of the trustee. Each has its own assets and liabilities. A claim by the trustee's private creditors is a debt of the private patrimony and is not to be met from trust funds. A claim by the beneficiary is a debt of the trust patrimony. To say that a beneficiary's personal right prevails over the rights of private creditors is therefore, on this view, a misperception. Neither right prevails over the other; rather, each is a right against a different patrimony. This analysis has found favor in Quebec, South Africa, and Scotland". Ver: Salience and Unity in the Mixed Jurisdiction Experience: Traits, Patterns, Culture, Commonalities: The Idea of Mixed Legal Systems. En: Tulane Law Review, Número 5, 2003, p. 115.

84 Esto es, un ente que, por ser destinatario directo de imputaciones juridicas, puede contratar, demandar, ser demandado, etc. 
representante legal que, en planos distintos, hagan todo lo necesario para que se obtenga la finalidad para la cual fue creado ese patrimonio. En el ámbito bursátil, hemos reconocido de manera positiva esta realidad en materia del fondo de garantía. En efecto, el artículo 1 de la Resolución Conasev No. 042-2006-EF/94.10, en su momento dispuso lo siguiente:

"El Fondo de Garantía es un patrimonio autónomo administrado por la Bolsa de Valores de Lima, cuya finalidad exclusiva es establecer un mecanismo de protección a favor de los comitentes que realicen operaciones en el mercado de valores (...)" (el énfasis es agregado).

Como se podrá advertir, la norma glosada expresamente establece que el patrimonio autónomo está sujeto, no a un régimen de propiedad u otro derecho real, sino a un régimen de administración. Por lo tanto, el gestor de ese patrimonio responde como lo hace el administrador de una sociedad anónima.

En el caso del Business Trust, el trustee es considerado un administrador con poderes legales. ${ }^{85}$ En el caso del fideicomiso de la industria financiera y de capitales, el fiduciario hace las veces de administrador y de representante legal. Lamentablemente, una concepción deforme de la realidad y pobre de los institutos legales involucrados en este fenómeno ("derecho", "patrimonio", etc.) sostiene la tendencia a representar al fiduciario de la referida industria como un sujeto que tiene un derecho real sobre los activos del fideicomiso y no como un sujeto que administra un patrimonio autónomo. Esta concepción se explica, en nuestra opinión, en la propensión que tenemos a explicar los nuevos fenómenos en función de categorías y esquemas pre-existentes; predisposición que demuestra nuestra aversión a invertir esfuerzo, tiempo e imaginación. Si, en lugar de copiar el modelo argentino del fideicomiso ${ }^{86}$, el legislador peruano hubiese estudiado la fuente de dicho modelo, se habría dado cuenta que, en materia bancaria y financiera, el Trust tenía correspondencia no con los "fenómenos reales" sino con los "fenómenos organizacionales". La tendencia de dicho legislador a emplear esquemas y categorías pre-existentes ha generado el resultado nocivo que denunciamos en este trabajo.

\section{RECONOCIENDO LA REALIDAD}

El fideicomiso contemporáneo presenta diversas funciones. En materia bancaria y financiera, el fideicomiso contemporáneo es empleado básicamente como un instrumento de gestión profesional de activos que tiene la ventaja comparativa de aislar los riesgos legales de las partes intervinientes al crear un patrimonio autónomo. $^{87}$

En el Perú, los fideicomisos más empleados son los fideicomisos bancarios y financieros, esto es, los fideicomisos creados con la finalidad de que un agente especializado gestione activos aislados de los patrimonios de las partes intervinientes. Ese agente debería tener la condición legal de administrador y representante del patrimonio autónomo, con poderes, funciones y responsabilidades similares a los que tienen los administradores de las sociedades anónimas. ${ }^{88}$

Los esquemas y las categorías deben servir para procesar la realidad, no para sustituirla, ni para deformarla. La tesis que equipara dominio

85 "The primary role of the trustee is to manage the funds of the trust. In managing those funds, the trustee must not only safeguard them from loss of value but must also invest the assets so as to produce income". Ver: PENNER, Stephen M., International Investment and the Prudent Investor Rule: the Trustee's Duty to Consider International Investment Vehicles. En: Michigan Journal of International Law, Número 16, 1995, p. 602.

86 Para mayor detalle: ESCOBAR, Freddy, Traditions (...), p. 190 y ss.

87 Ver: ESCOBAR, Freddy, Traditions (...), pp. $212-218$.

88 "The primary role of the trustee is to manage the funds of the trust. In managing those funds, the trustee must not only safeguard them from loss of value but must also invest the assets so as to produce income". Ver: PENNER, Stephen M., Luc. Cit. 
fiduciario con derecho real es incorrecta dadas las premisas con las que operamos tanto en al ámbito político-filosófico, como en el ámbito legal. La incorrección denunciada no hace otra cosa que colocar una máscara legal que atrae nuestra atención hacia cuestiones que, en términos comparativos, carecen de relevancia normativa, pues más importante que determinar cómo se inscribe el dominio fiduciario es determinar cómo han de regularse los problemas de agencia respecto de las labores del fiduciario, los regímenes de responsabilidad aplicables a esas labores, los esquemas de compensación del fiduciario, las posibilidades de modificación unilateral de los beneficios por parte del fideicomisario, etc.

La tesis que esquematiza y categoriza al fideicomiso como un tipo de derecho real no permite que nos enfoquemos en los problemas reales que el fideicomiso organizacional requiere enfrentar y resolver. Por lo tanto, esa tesis debe ser abandonada de inmediato por ser intrínsecamente ineficiente e incluso inmoral.

Síntomas positivos que sugieren que hemos empezado a esquematizar y categorizar correctamente la realidad pueden ser hallados en la Resolución Conasev No. 080-2008-EF/94.01.1 yen la Resolución Conasev No. 008-2009-EF/94.01.1. La primera aprueba el régimen aplicable a la administración temporal del fondo de garantía de la Bolsa de Valores de Lima, mientras que la segunda aprueba el procedimiento sancionador especial para ciertas denuncias de los comitentes contra las sociedades agentes de bolsa.

De acuerdo con el artículo 1 de la Resolución Conasev No. 080-2008-EF/94.01.1, la Comisión Nacional Supervisora de Empresas y Valores administrará temporalmente el patrimonio autónomo constituido por el fondo de garantía de la Bolsa de Valores de Lima, hasta que una entidad privada asuma, en calidad de fiduciario, la administración de dicho patrimonio autónomo. Por su parte, de acuerdo con el artículo 2 de la Resolución Conasev No. 008-2009-EF/94.01.1, los comitentes legitimados para presentar denuncias contra las sociedades agentes de bolsa son las personas naturales, las personas jurídicas y los patrimonios autónomos que tengan o hubieran tenido la condición de clientes.

Como se advierte, estas normas positivas indican expresamente que el fiduciario administra un patrimonio autónomo y que este último es un sujeto de derechos al igual que la persona natural y la persona jurídica. A partir de este reconocimiento correcto de la realidad, podemos comenzar a discutir el diseño de un régimen legal eficiente que enfrente los problemas más trascendentales que el fideicomiso presenta como forma organizacional: las manos muertas, los costos de agencia y los estándares de diligencia del fiduciario.

\section{VIII.CONCLUSIÓN}

A lo largo de estas páginas hemos demostrado cómo los esquemas y las categorías pueden influir negativamente en la comprensión y regulación de fenómenos legalmente relevantes que merecen una categorización y una esquematización propias.

El supuesto emblemático elegido para demostrar nuestra premisa ha sido el dominio fiduciario, debido a la importancia superlativa que el fideicomiso ha adquirido en la economía peruana en las últimas dos décadas, a pesar de la pobre e incorrecta regulación de que ha sido víctima en esta parte del continente.

Esta situación demanda que los abogados hagamos un esfuerzo por liberarnos de las categorías y esquemas clásicos que impiden una cabal comprensión de los fenómenos del mercado, imposibilitando una categorización y una esquematización que conduzcan a una regulación adecuada de estos fenómenos de acuerdo a su función económica. 